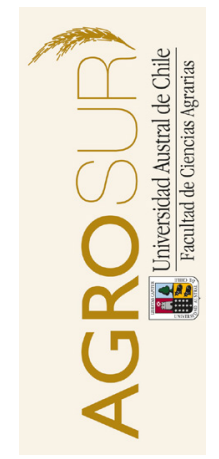

\title{
Utilización de Trichoderma spp. e hidróxido de cobre, como tratamiento foliar, para el control del tizón tardío de la papa (Phytophthora infestans)
}

\author{
Use of Trichoderma spp. and copper hydroxide, as a foliar treatment, for the \\ control of late blight of potato (Phytophthora infestans)
}

\author{
Andrade, N. ${ }^{a}$, Venegas, C. ${ }^{b}$, Cancino, B. ${ }^{a}$, Doussoulin, H. ${ }^{a *}$ \\ ${ }^{a}$ Instituto de Producción y Sanidad Vegetal, Facultad de Ciencias Agrarias, Universidad Austral de Chile. \\ ${ }^{b}$ Centro de Estudios Tecnológicos (CET), Chonchi, Chiloé.
}

\begin{tabular}{l} 
A R T I C L E I N F O \\
\hline Article history: \\
Received 22.03.2017 \\
Accepted 29.11.2017 \\
\hline Keywords: \\
Trichoderma \\
copper hydroxide \\
Phytophthora infestans \\
late blight \\
potato \\
\hline Original Research Article, \\
Crop Science \\
\hline *Corresponding author: \\
Nancy Andrade, \\
Herman Doussoulin \\
E-mail address: \\
nandrade@uach.cl, \\
hdoussoulin@gmail.com
\end{tabular}

\begin{abstract}
A B S T RA C T
Potato late blight, caused by Phytophtora infestans (Mont.) de Bary, is considered as the most crucial and devastating disease affecting this crop worldwide. In order to demonstrate the feasibility of employing applications of copper hydroxide fungicide (Kocide $2000 \AA$ ) and a mix of Trichoderma spp. on $P$. infestans control in potato crops in the Chiloé area, it was evaluated the in vitro and in vivo sensitivity of three strains of the Trichoderma genus (T. harzianum 1, T. harzianum 2, and T. viride) and P. infestans; and it was established the effective concentration 50 and 95 (EC50and EC95). Furthermore, it was estimated the area under the relative disease progress curve (rAUDPC), and the yield was determined. Trichoderma spp. strains were less sensitive than P. infestans to the fungicide. T. harzanium $1\left(\mathrm{EC}_{50} 174.6 \mathrm{mg} \mathrm{L}^{-1}\right.$ and $\left.\mathrm{EC}_{95} 1036.5 \mathrm{mg} \mathrm{L}^{-1}\right)$ and T. harzanium $2\left(\mathrm{EC}_{50} 158.6 \mathrm{mg} \mathrm{L}^{-1}\right.$ and $\left.\mathrm{EC}_{95} 912.4 \mathrm{mg} \mathrm{L}^{-1}\right)$ strains displayed similar behavior. $T$. viride was less sensitive $\left(\mathrm{EC}_{50} 581.1 \mathrm{mg} \mathrm{L}^{-1}\right.$ and $\mathrm{EC}_{95} 5052.2 \mathrm{mg} \mathrm{L}^{-1}$ ). P. infestans presented a higher sensibility with $\mathrm{EC}_{50} 6.52 \mathrm{mg} \mathrm{L}^{-1}$ and $\mathrm{EC}_{95} 17.2$ $\mathrm{mg} \mathrm{L}^{-1}$. Kocide $2000 \AA$ and Trichoderma spp. applications reduced the rAUDPC and maximized yields. These results demonstrate the importance of the disease management; furthermore, it suggests that Trichoderma and copper hydroxide improve crop health and yields.
\end{abstract}

\section{RESUMEN}

El tizón tardío de la papa causado por Phytophthora infestans (Mont.) de Bary, es considerada la enfermedad más importante y devastadora en este cultivo a nivel mundial. Con el objetivo de demostrar la factibilidad de utilizar aplicaciones del fungicida hidróxido de cobre (Kocide 2000®) y una mezcla de Trichoderma spp. en el control de P. infestans en el cultivo de la papa en Chiloé; se evaluó la sensibilidad in vitro e in vivo de tres cepas de Trichoderma (T. harzianum 1 y 2; T. viride) y P. infestans y se estableció la concentración efectiva 50 y 95 (CE50 y CE95), además se estimó el área bajo la curva relativa de progreso de la enfermedad (AUDPCr) y se determinó el rendimiento. Las cepas de Trichoderma spp. fueron menos sensibles que $P$. infestans al fungicida. Las cepas T. harzianum 1 (CE50=174,6 mg L-1 y CE95=1036,5 mg L-1) y T. harzianum 2 (CE50=158,6 mg L-1 y CE95=912,4 mg L-1) presentaron comportamiento similar, $T$. viride fue menos sensible (CE50=581,1 mg L-1 y CE95=5052,2 mg L-1). P. infestans presentó una mayor sensibilidad con CE50=6,52 mg L-1 y CE95=17,2 mg L-1. Aplicaciones de Kocide 2000® y Trichoderma spp. disminuyeron el AUDPCr y maximizaron los rendimientos. Los resultados demuestran la importancia del manejo de la enfermedad y además sugieren que aplicaciones de Trichoderma e hidróxido de cobre, favorecen la sanidad del cultivo y el rendimiento.

Palabras clave: Trichoderma, hidróxido de cobre, Phytophthora infestans, tizón tardío, papa.

\section{INTRODUCCIÓN}

La papa (Solanum tuberosum L.) es una de las principales especies cultivadas en Chile, ocupando una superficie aproximada de 53.000 ha y alcanzando rendimientos promedio de $218 \mathrm{qqm} \mathrm{ha}^{-1}$ (ODEPA, 2017), además, el Archipiélago de Chiloé ubicado en el sur de
Chile $\left(42^{\circ} 37^{\prime} \mathrm{S}, 73^{\circ} 54^{\prime} 30\right.$ ) es considerado un subcentro de origen de esta especie (Solano et al., 2007; López et al., 2015), utilizándose actualmente en esta zona, más de 200 variedades de papa, de diferentes formas, colores y sabores.

El tizón tardío de la papa causado por Phytophthora infestans (Mont.) de Bary, causa una de las enferme- 
dades más importantes y devastadoras del cultivo a nivel mundial (Hijmans et al., 2000). El desarrollo de la enfermedad se ve favorecido por humedades relativas sobre el $80 \%$ y temperaturas de entre 15 y $25{ }^{\circ} \mathrm{C}$ (Mizubuti y Fry, 2006). Este oomycete se caracteriza por afectar todas las partes de la planta, produciendo manchas necróticas en hojas, tallos y tubérculos (Schumann y D'Arcy, 2000). Se estima que el costo asociado a control y perdidas en la producción, es de USD \$ 6.7 billones anuales a nivel mundial (Runno-Paurson et al., 2013) y en Chile se han observado perdidas de rendimiento por sobre el 50\% (Acuña, 2011).

Para asegurar el éxito productivo del cultivo, los productores pueden llegar a realizar entre 10 a 15 aplicaciones de fungicidas por temporada, dependiendo de las condiciones ambientales (Riveros et al., 2003). Aunque los productos químicos presentan facilidades en su aplicación; su mala utilización ha generado problemas de resistencia por parte del fitopatógeno en Chile y el mundo (Davidse et al., 1981; McLeod et al., 2001; Riveros et al., 2003; Acuña et al., 2011). Estos problemas han conllevado a la búsqueda de nuevas alternativas de control que sean factibles de ser integradas en un plan de manejo integrado de esta enfermedad. El uso de control biológico a través de la utilización de microorganismos para el control del tizón tardío, es reconocido como una opción eficiente para su manejo (Quimby et al., 2002). Trichoderma es uno de los principales géneros de hongos utilizados en control biológico (Harman et al., 2004) y es considerado uno de los agentes más eficientes, debido a la capacidad de producir diferentes compuestos, tales como quitinasas, glucanasas y antibióticos (Harman et al., 2004) los que le otorgan diferentes mecanismos de acción que incluyen antibiosis, micoparasitismo e inducción de resistencia (Benítez et al., 2004; Harman et al., 2004; Harman, 2000). Su capacidad de control ha sido comprobada en diferentes patógenos como Rhizoctonia solani (Howell, 2003), Botrytis cinerea (Meyer et al., 1998), Alternaria solani (Fontenelle et al., 2011) e inclusive P. infestans (Yao et al., 2015).

La incorporación de microorganismos, en un plan de manejo integrado de la enfermedad, puede ser combinada con otras alternativas de control, como es la utilización de productos cúpricos, los cuales han sido utilizados y aseguran el éxito productivo en sistemas de producción orgánicos (Zarb et al., 2002). El ion cobre $\left(\mathrm{Cu}^{+2}\right)$ presenta acción multisitio, disminuyendo las posibilidades de generar resistencia por parte del patógeno y actúa a nivel celular, reaccionando con los grupos sulfhidrilos, hidróxidos amino y carboxílicos, inactivándolas y perturbando de esa forma la cadena respiratoria (McGrath, 2004; Agrios, 2005). Kocide ${ }^{\circledR}$ 2000, manufacturado por DuPont, es un fungicida, bactericida preventivo y de contacto, que ejerce su acción por contacto impidiendo la formación de las esporas del hongo, siendo su ingrediente activo el hidróxido de cobre $\left(\mathrm{Cu}(\mathrm{OH})_{2}\right)$.

En orden de integrar la utilización de estos métodos en un plan de manejo del tizón tardío, en este trabajo se investigó la efectividad de aplicaciones de hidróxido de cobre y Trichoderma spp., para el control de tizón tardío de la papa en la zona de Chiloé.

\section{MATERIAL Y MÉTODOS}

\section{Sensibilidad in vitro a Hidroxido de cobre.}

Se utilizaron tres cepas de Trichoderma spp., de T. harzianum (1), T. harzianum (2) y T. viride (1), proporcionadas por el Centro de Educación y Tecnología de Chiloé. El aislado de Phytophthora utilizado fue obtenido previamente desde hojas muestras de papa provenientes de la zona de Frutillar, Región de los Lagos $\left(41^{\circ} 07^{\prime} \mathrm{S}, 73^{\circ} 03^{\prime} 0\right)$. Para el aislamiento de P. infestans las hojas fueron desinfectadas con hipoclorito de sodio al $1 \%$ por 5 minutos y posteriormente transferidas a placas Petri conteniendo Agar Centeno (AC) (Caten y Jink, 1968) que se incubaron a $20^{\circ} \mathrm{C}$ hasta observar crecimiento del hongo.

La multiplicación del material biológico y pruebas de sensibilidad in vitro fueron realizadas en el Laboratorio de Fitopatología del Instituto de Producción y Sanidad Vegetal, de la Facultad de Ciencias Agrarias de la Universidad Austral de Chile en Valdivia.

La sensibilidad a hidróxido de cobre (Kocide 2000() fue determinada en base a la inhibición del crecimiento micelial en placas Petri de $9 \mathrm{~cm}$ de diámetro conteniendo $\mathrm{AC}$ en concentraciones crecientes de producto comercial de: $0,1,10,100,1000$ y $5000 \mathrm{ppm}$. Discos de $6 \mathrm{~mm}$ de diámetro de cada cepa de Trichoderma y $P$. infestans crecidas previamente en AC por 7 días, fueron dispuestos en el centro de placas Petri con medio AC que contenían las respectivas concentraciones del producto evaluado. Las placas fueron mantenidas en cámara de incubación a $24^{\circ} \mathrm{C}$. La inhibición del crecimiento micelial se determinó como porcentaje de inhibición del crecimiento radial del micelio con respecto al tratamiento control, hasta cuando el tratamiento control (AC + 0 ppm de Kocide $\left.2000^{\circledR}\right)$ ocupó por completo la placa (Trichoderma spp. 48 horas y $P$. infestans 148 horas).

\section{Evaluación in vivo}

El ensayo fue establecido en invernadero de polietileno durante la temporada 2014 / 2015, en la Estación Experimental del Centro de Educación y Tecnología (CET), ubicado en el sector Notuco, Chonchi, Chiloé,

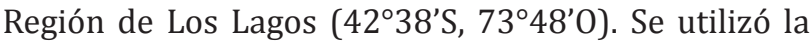
variedad de papa Murta (S. tuberosum cv. Murta), la que fue plantada en parcelas de 4 × 2,5 m, utilizando 
Cuadro 1. Tratamientos utilizados para la evaluación en campo de la efectividad de hidróxido de cobre (Kocide $2000 \circledR)$ y Trichoderma spp., en el control de tizón tardío (Phytophthora infestans), en papa (Solanum tuberosum) cv. Murta.

Table 1. Treatments used in the field evaluation of the effectiveness of copper hydroxide (Kocide 2000®) and Trichoderma spp., for the control of late blight (Phytophthora infestans), potato (Solanum tuberosum) cv. Murta.

\begin{tabular}{cccc}
\hline Tratamiento $\mathbf{N}^{\circ}$ & Tratamiento* & Dosis $\mathbf{C u}(\mathbf{O H})_{2}$ & Dosis Trichoderma spp. \\
\hline $\mathbf{1}$ & Control & 0 & 0 \\
$\mathbf{2}$ & Mezcla de Trichoderma spp. & 0 & $2 \mathrm{gr} \mathrm{L}^{-1}$ \\
$\mathbf{3}$ & $\mathrm{Cu}(\mathrm{OH})_{2}$ & $2 \mathrm{gr} \mathrm{L}^{-1}$ & 0 \\
$\mathbf{4}$ & Mezcla de Trichoderma spp. $+\mathrm{Cu}(\mathrm{OH})_{2}$ & $2 \mathrm{gr} \mathrm{L}^{-1}$ & $2 \mathrm{gr} \mathrm{L}^{-1}$ \\
\hline
\end{tabular}

un marco de plantación de $35 \mathrm{~cm}$ entre hilera y $65 \mathrm{~cm}$ sobre hilera, según los tratamientos dispuestos en el Cuadro 1.

Las plantas fueron inoculadas en dos ocasiones con P. infestans (inicio de tuberización y floración) con una suspensión que contenía $1,8 \times 10^{4}$ esporangios por $\mathrm{mL}$, hasta cubrir homogéneamente la planta. Los esporangios fueron obtenidos a partir de colonias del oomycete sembradas en AC durante 14 días a $18^{\circ} \mathrm{C}$. Para la obtención de la suspensión, $5 \mathrm{~mL}$ de $\mathrm{H}_{2} \mathrm{O}$ estéril fueron aplicados a cada placa, los que fueron recogidos por filtración en gasa estéril, en un matraz. Para la inoculación se utilizaron aspersores plásticos de $500 \mathrm{~mL}$ previamente esterilizados mediante luz UV.

Una mezcla en polvo de Trichoderma spp. (T. harzianum (1), T. harzianum (2) y T. viride (1), en partes iguales) conteniendo $1 \times 10^{10}$ unidades formadoras de colonias, fue utilizada en el ensayo en invernadero (proporcionada por el Centro de Educación y Tecnología (CET)). Al momento de plantación los tubérculos fueron sumergidos durante 30 minutos en 50 litros de una solución de la mezcla de Trichoderma spp., en dosis de $2 \mathrm{~g} \mathrm{~L}^{-1}$. Las aplicaciones de Trichoderma spp. y Kocide $2000 \AA$ fueron realizadas cada 2 semanas a partir de la emergencia del cultivo, mediante la utilización de bomba de espalda con dosis de $2 \mathrm{~g} \mathrm{~L}^{-1}$ de Trichoderma spp., considerando un mojamiento de $500 \mathrm{~L} \mathrm{ha}^{-1}$, se realizó un total de 7 aplicaciones durante la duración de este estudio.

Se evaluó el porcentaje de destrucción del follaje utilizando la metodología descrita por Henfling (1987). Las evaluaciones fueron realizadas cada dos días desde que se observaron los primeros síntomas de daño en el follaje, que corresponde a 80 días después de la plantación, extendiéndose por 84 días hasta la finalización del ensayo. Esta información fue utilizada para estimar el área bajo la curva de progreso de la enfermedad (AUDPC) (Ecuación 1) (Simko y Piepho, 2012).

$$
A U D P C=\sum_{i=1}^{n-1} \frac{Y_{i}+Y_{i+1}}{2} \times\left(t_{i+1}-t_{i}\right)
$$

En donde: AUDPC= área bajo la curva de progreso de la enfermedad, $Y_{i}=$ porcentaje de follaje dañado inicial, $Y_{i+1}=$ porcentaje de follaje dañado actual, $t_{i+1}$ =tiempo actual, $\mathrm{t}_{\mathrm{i}}=$ tiempo inicial $\mathrm{y} \mathrm{n}=$ número total de evaluaciones.

Posteriormente, se calculó el área relativa bajo la curva de progreso de la enfermedad (AUDPCr) (Formula 2).

$$
A U D P C r=\frac{A U D P C / 100}{t_{\text {final }}-t_{\text {inicial }}}
$$

En donde: AUDPCr= área relativa bajo la curva de progreso de la enfermedad, AUDPC= área bajo la curva de progreso de la enfermedad, $\mathrm{t}_{\text {final }}=$ tiempo en días de la última evaluación y $t_{\text {nicial }}=$ tiempo en días de la primera evaluación.

El rendimiento fue evaluado al momento de cosecha, en base al peso total de los tubérculos de las dos hileras centrales de cada parcela.

\section{Diseño experimental y análisis estadístico}

En el ensayo de sensibilidad in vitro las placas fueron dispuestas en un diseño en bloques completamente al azar con 5 repeticiones por tratamiento. El porcentaje de inhibición de cada cepa de Trichoderma y $P$. infestans fue analizado mediante ANOVA simple, y una prueba de separación de medias de Tukey $(\mathrm{p}<0,05)$.

Para obtener los valores de $\mathrm{CE}_{50}$ y $\mathrm{CE}_{95}$ de cada compuesto para cada cepa de Trichoderma y P. infestans, se realizó un análisis de regresión lineal, donde las dosis en $\mathrm{mg} \mathrm{L}^{-1}$ de las concentraciones de fungicidas fueron transformadas a $\log _{10}$ y el porcentaje de inhibición del microorganismo a grados PROBIT.

Para determinar la efectividad de los tratamientos en el ensayo in vivo, se utilizó un diseño en bloques completamente al azar con tres repeticiones por cada tratamiento.

Los datos de de AUDPCr y rendimiento fueron ana- 
lizados mediante un ANOVA simple y en caso de existir diferencias estadísticamente significativas se realizó una separación de medias mediante la prueba de Tukey $(p<0,05)$. Para el análisis de datos se utilizó el programa Stat Soft Statistica 7.0.

\section{RESULTADOS Y DISCUSIÓN}

\section{Sensibilidad in vitro.}

Todas las cepas de Trichoderma y P. infestans utilizadas en este ensayo mostraron diferentes porcentajes de inhibición del crecimiento micelial, al ser expuestos a diferentes dosis del fungicida Kocide $2000 \circledR$ (Cuadro 2), las cepas pertenecientes al género Trichoderma mostraron una menor sensibilidad al fungicida que $P$. infestans, T. harzianum 1 y 2 mostraron un comportamiento similar, inhibiéndose el micelio en un $100 \%$ y 97,1\% respectivamente, a concentraciones de $5000 \mathrm{mg} \mathrm{L}^{-1}$.
T. viride fue la cepa menos sensible de Trichoderma, al mostrar los menores porcentajes de inhibición en todas las concentraciones utilizadas. La cepa de P. infestans utilizada, resultó ser la más sensible, obteniéndose un $100 \%$ de inhibición con la concentración de $100 \mathrm{mg} \mathrm{L}^{-1}$.

Las pruebas de sensibilidad in vitro a Kocide $2000 \AA$ realizadas para determinar las $\mathrm{CE}_{50}$ y $\mathrm{CE}_{95}$ permitieron determinar que $T$. viride presentó valores de $\mathrm{CE}_{50}$ y $\mathrm{CE}_{95}$ mayores que el resto de las cepas evaluadas, con $581,1 \mathrm{mg} \mathrm{L}^{-1}$ y $5052,2 \mathrm{mg} \mathrm{L}^{-1}$ respectivamente, valores superiores a lo observado en las cepas de T. harzianum (Cuadro 3), en donde T. harzianum (1) presentó una $\mathrm{CE}_{50}$ y $\mathrm{CE}_{95}$ de $174,6 \mathrm{mg} \mathrm{L}^{-1}$ y 1036,5 respectivamente, mientras que en $T$ harzianum (2) su $\mathrm{CE}_{50} \mathrm{y} \mathrm{CE}_{95}$ fue de 158,6 $\mathrm{mg} \mathrm{L}^{-1}$ y 912,4 $\mathrm{mg} \mathrm{L}^{-1}$ respectivamente. La cepa de $P$. infestans evidenció la mayor sensibilidad al fungicida presentando valores de $\mathrm{CE}_{50} \mathrm{y} \mathrm{CE}_{95}$ de $6,52 \mathrm{mg} \mathrm{L}^{-1} \mathrm{y}$ $17,2 \mathrm{mg} \mathrm{L}^{-1}$ respectivamente.

Valarmathi et al. (2013), evaluó la sensibilidad de

Cuadro 2. Inhibición del crecimiento micelial (\%) de Trichoderma harzianum (1 y 2), T. viride y Phytophthora infestans, sometido a diferentes concentraciones $\left(0,5,10,50,100,1000,2000\right.$ y $\left.5000 \mathrm{mg} \mathrm{L}^{-1}\right)$ de hidróxido de cobre (Kocide 2000®).

Table 2. Inhibition of mycelial growth (\%) of Trichoderma harzianum (1 and 2), T. viride and Phytophthora infestans, subjected to different concentrations $\left(0,5,10,50,100,1000,2000\right.$ and $\left.5000 \mathrm{mg} \mathrm{L}^{-1}\right)$ of copper hydroxide (Kocide $\left.2000 \AA\right)$.

\begin{tabular}{lcrrrr}
\hline & \multicolumn{5}{c}{ Inhibición de crecimiento } \\
\cline { 2 - 6 } \multicolumn{1}{c}{ Cepas } & $\mathbf{1}$ & $\mathbf{1 0}$ & $\mathbf{1 0 0}$ & $\mathbf{1 0 0 0}$ & $\mathbf{5 0 0 0}$ \\
\cline { 2 - 6 } & $10,6 \mathrm{a}$ & $44,0 \mathrm{a}$ & $100,0 \mathrm{a}$ & $100,0 \mathrm{a}$ & $100,0 \mathrm{a}$ \\
P. infestans. & $0,0 \mathrm{~b}$ & $0,0 \mathrm{~b}$ & $0,0 \mathrm{~b}$ & $53,1 \mathrm{~b}$ & $82,3 \mathrm{~b}$ \\
Trichoderma viridae & $0,7 \mathrm{~b}$ & $4,9 \mathrm{~b}$ & $36,7 \mathrm{c}$ & $83,8 \mathrm{c}$ & $97,1 \mathrm{a}$ \\
Trichoderma harzianum (2) & $0,0 \mathrm{~b}$ & $0,0 \mathrm{~b}$ & $35,8 \mathrm{c}$ & $86,0 \mathrm{c}$ & $100,0 \mathrm{a}$ \\
Trichoderma harzianum (1) & & & & & \\
\hline
\end{tabular}

Cuadro 3.Ecuación de regresión, coeficiente de determinación $\left(\mathrm{R}^{2}\right)$, concentración efectiva $50\left(\mathrm{CE}_{50}\right)$ y concentración efectiva $95\left(\mathrm{CE}_{95}\right)$ de inhibición del micelio de Trichoderma harzianum (1 y 2), T. viride y Phytophthora infestans, por hidróxido de cobre (Kocide 2000®).

Table 3. Regression equation, coefficient of determination $\left(\mathrm{R}^{2}\right)$, effective concentration $50\left(\mathrm{CE}_{50}\right)$ and effective dose $95\left(\mathrm{CE}_{95}\right)$ of mycelial inhibition of Trichoderma harzianum (1 and 2), T. viride and Phytophthora infestans, by copper hydroxide (Kocide $2000 \AA)$.

\begin{tabular}{|c|c|c|c|c|}
\hline \multirow{2}{*}{ Cepas } & \multirow{2}{*}{ Regresión* } & $\mathbf{R}^{2}$ & $* * \mathbf{C E}_{50}$ & $* * \mathbf{C E}_{95}$ \\
\hline & & $\%$ & $\left(\mathrm{mg} \mathrm{L}^{-1}\right)$ & $\left(\mathrm{mg} \mathrm{L}^{-1}\right)$ \\
\hline T. harzianum 1 & $y=2,1269 x+0,1088$ & $\mathrm{R}^{2}=0,97$ & 174,6 & 1036,5 \\
\hline T. harzianum 2 & $y=2,1648 x+0,1095$ & $\mathrm{R}^{2}=0,98$ & 158,6 & 912,4 \\
\hline T. viride & $y=1,7517 x+0,0901$ & $\mathrm{R}^{2}=0,98$ & 581,1 & 5052,2 \\
\hline P. infestans & $y=3,9065 x+0,4655$ & $\mathrm{R}^{2}=0,90$ & 6,52 & 17,2 \\
\hline
\end{tabular}

* y = porcentaje PROBIT de inhibición miceliar; $x=\log _{10}$ Concentración de fungicida. ${ }^{* *}$ Dosis calculada en base a la ecuación de regresión. 
T. viride a diferentes concentraciones de hidróxido de cobre, determinando que el hongo es capaz de sobrevivir a concentraciones de $3000 \mathrm{ppm}$, concordando con lo observado en este estudio, en donde T. viride fue capaz de crecer a 5000 ppm.

Los resultados obtenidos sugieren que las cepas de Trichoderma utilizadas son menos susceptibles al hidróxido de cobre (Kocide $2000 \AA$ ) que P. infestans, lo que permite inferir una posible aplicación conjunta de Kocide $2000 ®$ y Trichoderma para el control de tizón tardío en papa.

La tolerancia de Trichoderma spp. a cobre ha sido descrita por Vinale et al. (2004) quienes evaluaron la compatibilidad de T. harzianum (T22) y T. atroviride (P1) con oxicloruro de cobre, determinando un alto nivel de tolerancia que varió entre 21 y 1100 ppm.

Nuestros resultados indican que T. viride fue la cepa más tolerante a hidróxido de cobre, estudios realizados por Anand et al. (2005), evaluaron la interacción T. viride con cobre, determinando que la bioacumulación de Cu (II) en la superficie de la pared celular, sería el mecanismo utilizado para tolerar este metal, lo que explicaría la baja susceptibilidad de la cepa de T. viride observada en este estudio.

\section{Evaluación de campo}

\section{Incidencia y Severidad}

El efecto de los tratamientos de Kocide 2000® y Trichoderma sobre el nivel de daño de $P$. infestans es presentado a través del área bajo la curva de progreso de la enfermedad relativa (AUDPCr) (Figura 1). Se determinó la existencia de diferencias estadísticamente significativas para el AUDPCr entre tratamientos (Anova simple $\left.\mathrm{F}_{(3)}: 4,1078 ; \mathrm{p}<0,05\right)$. Los resultados muestran que los tratamientos de Trichoderma, Kocide 2000@ y su aplicación en conjunto produjeron una disminución significativa del AUDPCr en relación al tratamiento sin aplicación.

Los valores de AUDPCr presentan valores bajo 0,2 lo que refleja un bajo nivel de infestación en los distintos tratamientos incluido el control, sin embargo, las diferencias observadas permiten evidenciar un efecto protector de los tratamientos frente al daño producido por P. infestans en el cultivo de papa cv. Murta.

El rendimiento presentó diferencias estadísticamente significativas tratamiento (Anova simple $\mathrm{F}_{(3)}$ : 13,6393; p <0,05). Se puede apreciar (Figura 2) que la aplicación de Kocide $2000 ®$ y su aplicación en conjunto con Trichoderma permitieron aumentar los rendimientos del cultivo de papa en relación al tratamiento control, en donde el mayor rendimiento fue obtenido por el tratamiento de Trichoderma más hidróxido de cobre con aproximadamente 21 Ton ha ${ }^{-1}$.

La utilización de cobre para el control de enferme-

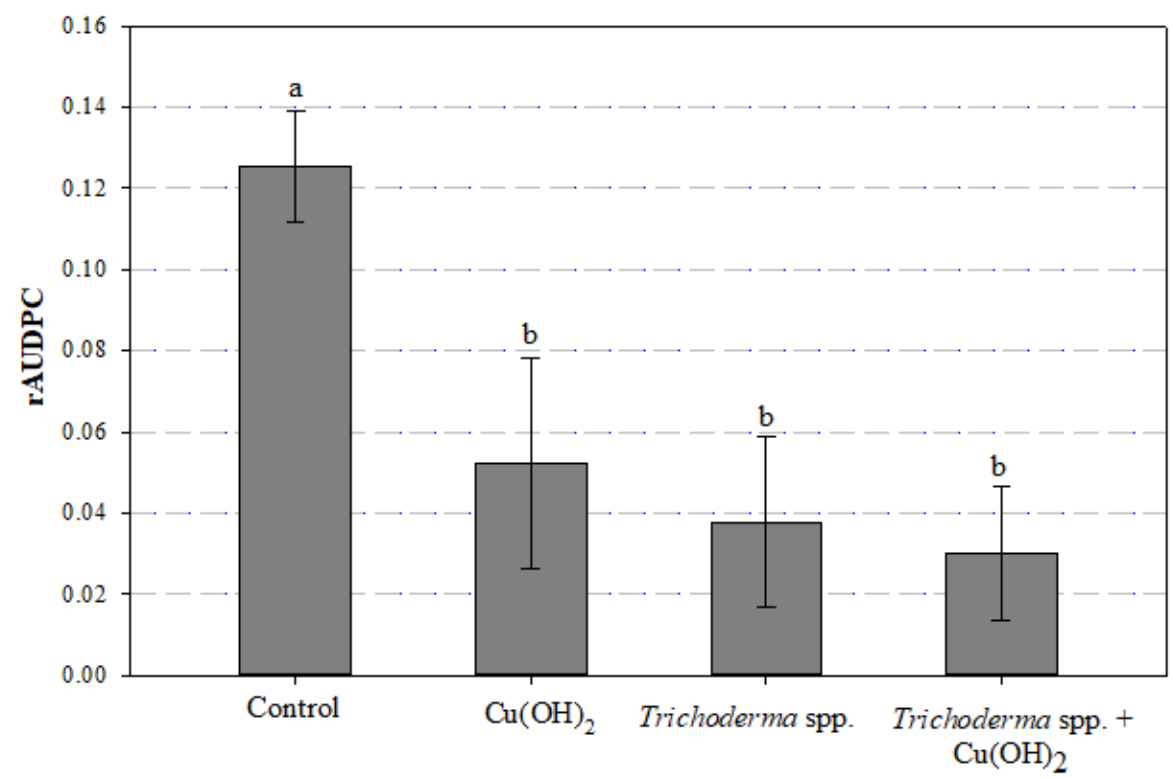

Letras diferentes en cada barra indica diferencias estadísticas entre tratamientos según test de Tukey HSD ( $\mathrm{p} \leq 0,05)$.

Figura 1. Área relativa bajo la curva (AUDPCr), de papa cv. Murta sometida a aplicaciones de hidróxido de cobre $\left(\mathrm{Cu}(\mathrm{OH})_{2}\right)$ y Trichoderma spp., para el control de tizón tardío (Phytophthora infestans), en cultivo bajo invernadero.

Figure 1. Relative area under the curve (AUDPCr) of potato cultivar "Murta" subjected to copper hydroxide $\left(\mathrm{Cu}(\mathrm{OH})_{2}\right)$ and Trichoderma spp. applications for late blight control (Phytophthora infestans) under greenhouse cultivation. 


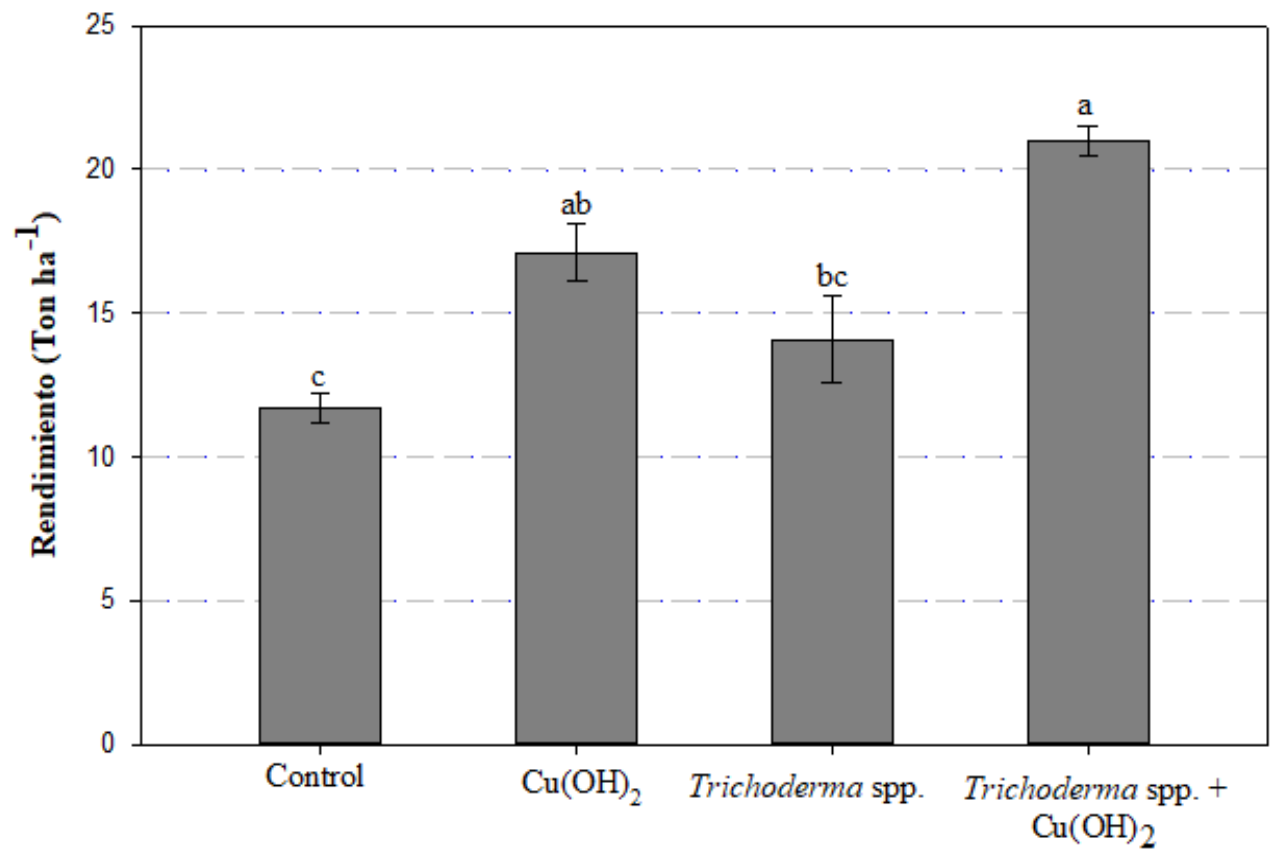

Letras diferentes en cada barra indica diferencias estadísticas entre tratamientos según test de Tukey HSD (p $\leq 0,05$ ).

Figura 2. Rendimiento (Ton ha $\left.{ }^{-1}\right)$ de papa cv. Murta, sometida a aplicaciones de hidróxido de cobre $\left(\mathrm{Cu}(\mathrm{OH})_{2}\right)$ y Trichoderma spp., para el control de tizón tardío (Phytophthora infestans), en cultivo bajo invernadero.

Figure 2. Yield (Ton ha-1) of potato cv. Murta, with copper hydroxide $\left(\mathrm{Cu}(\mathrm{OH})_{2}\right)$ and Trichoderma spp. applications for late blight control (Phytophthora infestans), under greenhouse cultivation.

dades en sistema de producción orgánico, constituye uno de los métodos más efectivos de control (Dorn et al., 2007), sin embargo, se puede agregar que también constituye una alternativa para sistemas de agricultura convencional. Disminuciones en la severidad y aumentos en el rendimiento similares a los observados en este estudio han sido reportados en evaluaciones realizadas por Dorn et al. (2007), quienes determinaron un efecto positivo sobre la disminución de la severidad de tizón tardío y el rendimiento en papa, reduciendo el porcentaje de hoja dañada desde un 23 a un $77 \%$ e incrementos en el rendimiento de 2 a 28\% (Dorn et al., 2007).

El género Trichoderma ha sido utilizado exitosamente en el control de diferentes enfermedades a nivel mundial (Harman, 2000; Sharon et al., 2001; Howell, 2003;Yao et al., 2015). La combinación de Trichoderma con fungicidas químicos ha demostrado un efecto positivo sobre la sanidad de los cultivos. Harman et al. (1996) determinaron que el uso de T. harzianum o en combinación con Iprodione produjo una reducción significativa en la severidad de la pudrición del racimo en vid. Al-Mughrabi (2008) determinó reducciones en la severidad de tizón tardío de un 27 y 36\% al utilizar T. atroviridae solo o en combinación con clorotalonil, respectivamente. El efecto positivo de aplicaciones de Trichoderma sobre parámetros de rendimiento ha sido también observado por Yao et al. (2015), quienes determinaron que el aislado de Trichoderma HNA14 redujo significativamente el índice de severidad de tizón tardío y aumentó la altura del tallo de la planta y el peso fresco y seco en cultivo de papa.

Los resultados obtenidos en este estudio determinaron un efecto positivo de la aplicación de hidróxido de Cobre y Trichoderma sobre el rendimiento, sugiriendo que aplicaciones de Trichoderma y Kocide $2000 \AA$ favorecen la sanidad del cultivo y el rendimiento, lo que permite contar con una posible nueva alternativa para disminuir las pérdidas que afectan la producción del cultivo de la papa, sin embargo, es necesario realizar nuevas evaluaciones a nivel de campo conducentes a determinar la efectividad del hidróxido de cobre y las cepas de Trichoderma spp., bajo diferentes condiciones ambientales, dosis de producto y una presión natural de la enfermedad.

\section{CONCLUSIONES}

Los resultados obtenidos en este estudio permitieron demostrar que las cepas de Trichoderma harzianum 1 y 2, T. viride y Phytophthora infestans, fueron sensibles a hidróxido de cobre (Kocide $2000 \AA$ ), siendo la cepa de $P$. infestans utilizada la más sensible. 
Existe compatibilidad entre aplicaciones conjuntas de hidróxido de cobre y Trichoderma spp. para el control de tizón tardío de la papa.

También, se concluye que aplicaciones de Kocide $2000 ®$ y Trichoderma presentaron un efecto positivo sobre la sanidad del cultivo, lo que favoreció el rendimiento.

\section{AGRADECIMIENTOS}

Proyecto FIA PYT-2012-0100, Centro de Educación y Tecnología (CET).

\section{REFERENCIAS}

Acuña, I., Sagredo, B., Gutierrez, M., Sandoval, C., Fahrenkog, A., Secor, G., Rivera, V., Mancilla, S., 2011. Characterization of Phytophthora infestans population in Chile. Presented at the Thirteenth EuroBlight Workshop, St. Petersburg, Russia, pp. 145-150.

Agrios, G., 2005. Plant Pathology. 5a ed. Academic Press, San Diego.

Al-Mughrabi, K. 2008. Biological Control of Phytophthora infestans of Potatoes using Trichoderma atroviride. Pest Technology 2, 104-108.

Anand, P., Isar, J., Saran, S., Saxena, R.K., 2006. Bioaccumulation of copper by Trichoderma viride. Bioresource Technology 97, 1018-1025.

Benítez, T., Rincón, A., Limón, M., Codón, A., 2004. Biocontrol mechanisms of Trichoderma strains. International Microbiology 7, 249-260.7

Caten, C., Jinks, J., 1968. Spontaneous variability of single isolates of Phytophthora infestans. I. Cultural variation. Canadian Journal of Botany 46(4), 329-348.

Davidse, L., Looijen, D., Turkensteen, L., Wal, D., 1981. Occurrence of metalaxyl-resistant strains of Phytophthora infestans in Dutch potato fields. Netherlands Journal of Plant Pathology 87, 65-68.

Dorn, B., Musa, T., Krebs, H., Fried, P., Forrer, H., 2007. Control of late blight in organic potato production: evaluation of copper-free preparations under field, growth chamber and laboratory conditions. European Journal of Plant Pathology 119(2), 217-240.

Fontenelle, A., Guzzo, S., Lucon, C., Harakava, R., 2011. Growth promotion and induction of resistance in tomato plant against Xanthomonas euvesicatoria and Alternaria solani by Trichoderma spp. Crop Protection 30, 1492-1500.

Harman, G.E., 2006. Overview of mechanisms and uses of Trichoderma spp. Phytopathology 96, 190-194.

Harman, G. 2000. Myths and dogmas of biocontrol changes in perceptions derived from research on Trichoderma harzinum T-22. Plant Disease. 84, 377-393.

Harman, G., Howell, C., Viterbo, A., Chet, I., Lorito, M., 2004. Trichoderma species--opportunistic, avirulent plant symbionts. Nature Review Microbiology. 2, 43-56.

Harman, G., Latorre, B., Agosin, E., San Martín, R., Riegel, D., Nielsen, P., Tronsmo, A., Pearson, R.C., 1996. Biological and integrated control of botrytis bunch rot of grape using trichoderma spp. Biological Control 7, 259-266.

Henfling, J., 1987. Late blight of potato Phytophthora infes- tans. Technical information Bulletin 4. International Potato Center, CIP, Lima, Perú. 25 pp.

Hijmans, R.J., Forbes, G.A., Walker, T.S., 2000. Estimating the global severity of potato late blight with GIS-linked disease forecast models. Plant Pathology. 49, 697-705.

Howell, C.R., 2003. Mechanisms Employed by Trichoderma Species in the Biological Control of Plant Diseases: The History and Evolution of Current Concepts. Plant Disease 87(1), 4-10.

López, M., Riegel, R., Lizana, C., Behn, A., 2015. Identification of virus and nematode resistance genes in the Chilota Potato Genebank of the Universidad Austral de Chile. Chilean Journal of Agricultural Research. 75, 320-327.

McGrath, M.T., 2004. What are Fungicides? Plant Health Instructor. http://www.apsnet.org/edcenter/intropp/ topics/Pages/fungicides.aspx (acceso, 13.04.2017).

McLeod, A., Denman, S., Sadie, A., Denner, F., 2001. Characterization of South African Isolates of Phytophthora infestans. Plant Disease 85, 287-291.

Meyer, G., Bigirimana, J., Elad, Y., Höfte, M., 1998. Induced systemic resistance in Trichoderma harzianum T39 biocontrol of Botrytis cinerea. European Journal of Plant Pathology 104, 279-286.

Mizubuti, E., Fry, W., 2006. Potato late blight, in: Cooke, B.M., Jones, D.G., Kaye, B. (Eds.), The Epidemiology of Plant Diseases. Springer, Netherlands, pp. 445-471.

Oficina de Estudios y Políticas Agrarias (ODEPA), 2017. http://www.odepa.cl/estadisticas/productivas/ (acceso, 13.04.2017).

Quimby, P.C., King, L.R., Grey, W.E., 2002. Biological control as a means of enhancing the sustainability of crop/land management systems. Agriculture Ecosystem \& Environment 88, 147-152.

Riveros, F., Sotomayor, R., Rivera, V., Secor, G., Espinoza, B., 2003. Resistencia de Phytophthora infestans (Montagne) de Bary a metalaxil, en cultivo de papas en el norte de Chile. Agricultura Técnica 63, 117-124.

Runno-Paurson, E., Williams, I., Metspalu, L., Kaart, T., Mänd, M., 2013. Current potato varieties are too susceptible to late blight to be grown without chemical control under North-East European conditions. Acta Agriculturae Scandinavica, Sect. B - Soil Plant Science 63, 80-88.

Schumann, G., D'Arcy, C., 2000. Late blight of potato and tomato. Plant Health Instructor. http://www.apsnet.org/ edcenter/intropp/lessons/fungi/Oomycetes/Pages/ LateBlight.aspx (acceso, 13.04.2017).

Sharon, E., Bar-Eyal, M., Chet, I., Herrera-Estrella, A., Kleifeld, O., Spiegel, Y., 2001. Biological Control of the Root-Knot Nematode Meloidogyne javanica by Trichoderma harzianum. Phytopathology 91, 687-693.

Simko, I, Piepho, H., 2012. The area under the disease progress stairs: calculation, advantage, and application. Phytopathology 102(4), 381-9.

Solano, J., Morales, D., Anabalón, L., 2007. Molecular description and similarity relationships among native germplasm potatoes (Solanum tuberosum ssp. tuberosum L.) using morphological data and AFLP markers. Electronic Journal of Biotechnology. 10, 436-443.

Vinale, F., D’Ambrosio, G., Abadi, K., Scala, F., Marra, R., Turra, D., Woo, S., Lorito, M. 2004. Application of Trichoderma harzianum (T22) and Trichoderma atroviride (P1) as 
plant growth promoters, and their compatibility with copper oxychloride. Journal of Zhejiang University Science $30,2-8$

Yao, Y., Li, Y., Chen, Z., Zheng, B., Zhang, L., Niu, B., Meng, J., Li, A., Zhang, J., Wang, Q., 2015. Biological Control of Potato Late Blight Using Isolates of Trichoderma. American Journal of Potato Research 93, 33-42.
Zarb, J., Ghorbani, R., Juntharathep, P., Shotton, P., Santos, J., Wilcockson, S., Leifert, C., Litterick, A. M., Bain, R. A., Wolfe, M., 2002. Control strategies for late blight in organic potato production, in: Proceedings of the UK Organic Research 2002. Conference Organic Centre Wales, Institute of Rural Studies, University of Wales Aberystwyth, pp. 221-222. 